\title{
How can Sodium MRI techniques help us understand acute stroke?
}

\author{
Andrew J Fagan
}

Centre for Advanced Medical Imaging, St. James's Hospital and School of Medicine, Trinity College, University of Dublin, Ireland

Tel: $+353-18964331$

Fax: $+353-14103478$

Email: andrew.fagan@tcd.ie

\section{SUMMARY}

This article addresses the potential usefulness of sodium MRI in the acute phase of stroke, asking whether the additional time required to acquire high quality sodium images is justified in the time-critical minutes following the presentation of a patient with symptoms. It begins with a description of the pathophysiology of stroke and the implications of the increasing bioenergetic failure on the sodium content in tissue. Recent studies which have aimed at imaging the subtle changes in this sodium content in stroke patients are then reviewed, followed by experiments in animal models of stroke which circumvent some of the limitations of the human studies. Finally, steps which will likely be required to translate these latest model findings into human studies are discussed, including new MR imaging techniques which may provide a boost in signal and allow for the introduction of relaxationtime contrast and quantification of the sodium concentration.

Keywords: sodium-MRI, stroke, penumbra, sodium-perfusion mismatch Sodium quantification, TSC. 


\section{EXECUTIVE SUMMARY}

\section{Background}

- Considerable progress has been made in the field of Na-MRI over the past decade, such that reasonable quality brain images can now be obtained using clinical 3T scanners with 10 minute acquisition times;

\section{Link Between Sodium and Stroke}

- From a stroke perspective, sodium MRI may offer a rapid, non-invasive method of identifying patients suitable for a therapeutic intervention;

- Changes in the intra-cellular sodium concentration are known to occur shortly after an ischaemic insult due to the impaired functioning of the $\mathrm{Na}^{+} / \mathrm{K}^{+}$APTase, which is responsible for maintaining cellular homeostasis;

\section{Clinical and Pre-Clinical Stroke Na-MRI Studies}

- Several studies in both animal models of stroke and in stroke patients have detected this change in sodium imaging experiments, with early increases observed in core, infarcted tissue from an early time-point after stroke onset;

- Evidence in animal models suggests that the sodium signal averaged across all tissue compartments may, in fact, decrease in penumbra tissue in the acute stroke phase, potentially offering a method of detecting penumbra tissue, i.e. underperfused tissue which is at risk of infarction but still amenable to salvage;

\section{Scope for Improvement}

- Continuing improvements in image quality through hardware and imaging pulse sequence developments are opening up many new possibilities, for example making it possible to accurately quantify the tissue sodium concentration (and perhaps also the intra-cellular compartment) and moving beyond mere sodium spin-density weighted imaging with the introduction of relaxation-time contrast. 


\section{FUTURE PERSPECTIVE}

It can be expected that the next 5-10 years will see the rapid pace of developments in the field of Na-MRI continue, with further improvements in the achievable SNR per unit time enabling accurate quantification of the tissue sodium concentration at better spatial resolutions in the human brain. The adoption by Na-MRI of new ideas in compressed sensing and iterative reconstruction coupled with developments with radiofrequency coil technology may realise this improvement. Relaxation-time changes are likely to occur in ischemic tissue resulting from intra- and extra-cellular compartmental shifts, and it is anticipated that relaxation-time contrast or indeed sodium relaxometry will yield further insights into the usefulness of Na-MRI in acute stroke imaging. The use of multiple quantum coherence techniques which attempt to separate the signal from the intra- and extra-cellular compartments will likewise benefit from SNR increases and further work in this area can be expected. Additional clinical studies involving acute stroke patients are required to inform on the usefulness of Na-MRI in this time-critical period, which will be aided by the growing availability of Na-MRI techniques.

\section{INTRODUCTION}

The aim of this article is to provide a critical overview of the current use of sodium MRI in the acute phase of stroke, focussing initially on the relatively limited studies conducted to date in humans and then moving on to describe the more numerous studies which have been carried out in animal models of stroke which, despite the inherent limitation of such models particularly when translating findings from them to the human system, nevertheless may indicate areas for future focus in human studies. It can be argued that, despite the drastically more complicated cerebral vascular system in the human compared to that in, say, the rat brain, at a cellular level both human and rat brain systems are not so dissimilar, and cellular bioenergetic failure and the consequent change in sodium signal detectable via MRI can thus be expected to manifest in both systems in a similar manner. The main difference is in the time at which the bioenergetic failure occurs in brain regions downstream from a blockage: in rat models, most of the damage occurs relatively quickly, with the stroke lesion reaching its maximum extent within 4 to 6 hours after the stroke was induced. In the human brain, by contrast, the vastly more sophisticated vascular system which incorporates a higher degree of 
redundant blood supply to most areas (the so-called collateral supply), the situation is more complicated with a slower evolution of the stroke lesion extending out to 48 hours or more, suggesting a lengthened time-window in which to intervene therapeutically. Thus changes in the sodium MR signal observed in animal models, whether due to changes in sodium concentration or perhaps relaxation-time related signal changes specifically introduced into the images, should be translatable into human studies. This article sets out the current stateof-the-art in this area, and highlights the challenges which will need to be overcome to allow for routine clinical imaging of acute stroke patients.

\section{BACKGROUND}

After attempts to do sodium MRI in the 1980's met with limited success, the field is currently enjoying a renaissance driven primarily by technological developments in MR technology which are making it possible to explore the many possible clinical applications of Na-MRI, some of which have been postulated since the early days of MRI but have heretofore remained tantalisingly beyond reach. Indeed, $\mathrm{Na}$ spin density-weighted images with reasonable signal to noise ratio (SNR) and spatial resolution can now be obtained in the brain in less than 10 minutes using standard $3 \mathrm{~T}$ clinical imaging platforms ${ }^{(1)}$, and a growing number of groups worldwide are working to push the boundaries of this exciting technique ever further. Examples of clinical application areas which have been investigated to date include cerebral stroke ${ }^{(2,3,4)}$, brain tumour ${ }^{(5,6)}$, breast tumour ${ }^{(7)}$, cardiac infarction ${ }^{(8)}$, Alzheimer's disease ${ }^{(9)}$, multiple sclerosis ${ }^{(10)}$, kidney ${ }^{(11,12)}$, osteoarthritis in cartilage ${ }^{(13,14)}$, while the first whole body sodium images were recently presented ${ }^{(15)}$.

In addition, considerable effort is going into the extension of the Na-MRI technique beyond mere spin-density weighting, to explore the introduction of relaxation-time contrast and indeed relaxometry mapping, and the use of multiple quantum filter techniques to separate intra- from extra-cellular sodium compartments in tissue, while future developments may well involve $\mathrm{Na}$ diffusion weighted imaging and beyond. The resurgence of Na-MRI has come about primarily as a result of a maturation of MR technology, encompassing the availability of high field strength systems $(\geq 3 \mathrm{~T})$, improvements in system electronics and radiofrequency coil design concepts, and advanced imaging pulse sequences which combine 
rapid acquisitions with high sensitivity to the short $\mathrm{T}_{2}$-relaxation time components of sodium nuclei encountered in vivo.

The change in sodium concentration in infarcted tissue, that is tissue which has died following bioenergetic failure due to the interruption of blood supply to the area, typically following a stroke, has been known for many years, and indeed early attempts to study both cerebral and cardiac infarction in humans via Na-MRI were largely proof-of-concept and revealed little of use for acute patient imaging. In more recent years, a growing number of studies in pre-clinical animal models of cerebral stroke have been reported in the literature, to the level where a picture of the potential usefulness of Na-MRI for acute stroke imaging is now emerging. The critical role of sodium ions in maintaining healthy cellular function, followed by the physiological link between sodium concentration and infarction, is outlined in the next section

\section{LINK BETWEEN SODIUM AND STROKE}

The potential of Na-MRI for many disease investigations in underpinned by the critical role of sodium in many cellular functions such as the regulation of mitosis, cellular proliferation, the mediation of sodium / calcium exchange, and energy metabolism via the $\mathrm{Na}^{+} / \mathrm{K}^{+}$ATPase pump function located in the cell membrane. Indeed, the large sodium ion concentration gradient across the cell membrane established by the $\mathrm{Na}^{+} / \mathrm{K}^{+}$ATPase, which maintains the intra-cellular concentration at approximately $10 \mathrm{mmol} / \mathrm{L}$ and the extra-cellular sodium concentration at approximately $140 \mathrm{mmol} / \mathrm{L}$, is essential for the generation and propagation of action potentials, cell volume regulation and other cellular homeostatic and regulatory functions. Disease processes which alter this energy-dependent pump function will thus lead to an altered tissue sodium content, which may be detected via Na-MRI techniques.

The mortality and morbidity associated with stroke are well documented: it is the third leading cause of death and the leading cause of disability in developed countries ${ }^{(16)}$. Despite much research in the area of neuroprotectants and therapies, only one approved treatment currently exists, intravenous thrombolysis with recombinant tissue plasminogen activator (rtPA). However, it is only approved for administration within 4.5 hours of symptom onset, a time-window based on epidemiological studies of large cohort of patients, which effectively excludes its use in many patients for whom the time from onset is unknown or exceeds the 
time-window (although intrarterial thrombolysis out to 6 hours is currently performed in many institutions). Its use is further restricted due to other factors such as the risk (7\%) of intracranial haemorrhage associated with $\mathrm{rtPA}^{(16)}$, such that the estimated uptake of rtPA is less than $10 \%$ in stroke patients ${ }^{(17)}$. It is likely that a more tailored approach to the use of this treatment in individual patients would be appropriate, for example allowing for an extension of the time window during which it could be administered, and indeed recent studies have suggested that there may be benefit to using rtPA out to 6 hours ${ }^{(18)}$. However, such a tailored approach requires a reliable method for assessing the potential for benefit of rtPA to an individual, and it is here that Na-MRI, coupled with other MRI techniques, may have a role to play.

Ischaemic stroke is a catastrophic event in which the blood supply to a part of the brain is restricted and oxygen and glucose supply to cells within the affected region is severely limited. The loss of nutrient supply to cells in turn compromises energy metabolism within the stroke-affected area, leading to a rapid loss in ATP and hence in ionic homeostasis. One immediate consequence of this is a reduced efficiency of the $\mathrm{Na}^{+} / \mathrm{K}^{+}$pump function and a resulting increase in the intra-cellular sodium concentration. This in turn leads to oedema and eventually to cell membrane rupture. To stratify patients who might benefit from rtPA treatment, conventional MRI techniques employed in the acute phase of stroke have attempted to identify the presence of so-called "penumbral" tissue regions which are hypoperfused yet still viable using the so-called "perfusion-diffusion mismatch", wherein the mismatch between the ischaemically-injured tissue and hypoperfused tissue is determined from diffusion- and perfusion-weighted ${ }^{1} \mathrm{H}-\mathrm{MRI}$ (DWI and PWI) respectively. Tissue in such penumbra regions are at risk of infarction, but can be salvaged by a timely reperfusion of the affected tissue, hence the presence of such at-risk tissue in a stroke patient would give confidence for a potential benefit for the administration of rtPA.

The DWI technique is sensitive to the molecular diffusion of water molecules in tissue, and indeed can be used to obtain a quantitative estimate of the diffusion coefficient (the "apparent diffusion coefficient", or ADC). The cytotoxic oedema which occurs within minutes of the ischaemic onset results in the restriction of the diffusion of water molecules within the affected tissue, which can be easily detected in an ADC map. The PWI technique tracks a bolus of paramagnetic contrast agent as it perfuses through tissue and provides a semiquantitative measure of the regional cerebral blood flow, and thus may be used to determine 
the degree of underperfusion caused by the blockage. However, despite early optimism relating to the perfusion-diffusion mismatch and studies which have shown that thrombolytic treatment outcomes are improved in patients selected based on an MRI diagnosis ${ }^{(19)}$, it is now clear that this approach leads to a lack of precision in the separation of still-viable from irreversibly damaged ischaemic core tissue. For example, large deviations have been observed between areas with decreased ADC compared to infarct determined by the gold standard technique of histology after stroke ${ }^{(20)}$ while ADC values have also been observed to normalize in areas of permanently damaged tissue during the sub-acute phase ${ }^{(21)}$, suggesting that the lesion delineated by DWI does not provide a definitive indicator of infarcted tissue in the acute stroke phase. Furthermore, definitive thresholds of blood flow for penumbra or infarct have not been identified, and hence the area of hypoperfusion revealed by PWI may overestimate the volume of tissue which is realistically "at-risk", due to the inclusion of areas of benign oligemia. Overcoming this problem will require the establishment of such thresholds, although it is known that they depend on the duration of ischemia and any reperfusion which has occurred, whether thrombolysis-induced or spontaneous ${ }^{(22)}$. New approaches to the analysis of perfusion-weighted data may moderate the severity of the overestimation $^{(23)}$.

The Na-MRI technique provides a measure of the tissue sodium concentration (TSC), which represents an average of the sodium concentration in the intra- and extra-cellular tissue compartments weighted by their respective volume fractions. For example, in brain tissue, the TSC is typically $45 \mathrm{mmol} / \mathrm{L}$, considering an intra-cellular volume fraction of approximately $70 \%$ and an extra-cellular volume fraction of $30 \%$ (the latter comprising a 5\% vascular space and a 25\% extra-cellular extra-vascular space) Following an ischemic insult to the tissue and the failure of the $\mathrm{Na}^{+} / \mathrm{K}^{+}$pump, the intra-cellular sodium concentration gradually increases above its nominal 10-15 $\mathrm{mmol} / \mathrm{L}$ level, while the extra-cellular concentration, buffered by the body, is maintained at $140 \mathrm{mmol} / \mathrm{L}$. This result in a net increase in the TSC, and this has been measured in both animal model studies ${ }^{(24,25,26,27,28)}$ and human stroke patients ${ }^{(29,3,4,2)}$. 


\section{CHALLENGES WITH IMAGING OF SODIUM NUCLEI}

The difficulty with Na-MRI lies primarily with the lower concentration and MR-sensitivity of sodium nuclei compared to hydrogen nuclei in vivo, resulting in signal levels approximately 20,000 times lower than that obtained using conventional ${ }^{1} \mathrm{H}-\mathrm{MRI}$ techniques. In addition, the resonant frequency of sodium nuclei is approximately one quarter that of hydrogen nuclei, and hence broadband MRI systems with dedicated radiofrequency (RF) transmit amplifiers and RF coils for transmission and detection of the sodium signal are also required; although available for clinical 3T systems, at present these hardware options are not typically installed as standard. Indeed, the development of new RF coil designs has contributed to the increased SNR achievable in Na-MRI experiments and remains an active area of research. For human brain imaging, transceiver volume resonators based on a birdcage design are typically used; examples include single-tuned resonators for ${ }^{23} \mathrm{Na}$ frequencies only, necessitating a swop of the RF coils to acquire ${ }^{1} \mathrm{H}$ images ${ }^{(6,3)}$, or dual-tuned to allow for ${ }^{1} \mathrm{H}$ - and ${ }^{23} \mathrm{Na}$ imaging without disturbing the patient in the scanner and losing valuable time ${ }^{(30,31)}$. However, such dual coil configurations invariably compromise the SNR on one channel, usually selected to be the ${ }^{1} \mathrm{H}$ channel, and the effect this may have on the quality of the images typically acquired as part of a clinical stroke protocol, for example involving time-of-flight angiography or echo planar imaging-based diffusion and perfusion measurements, have not been reported.

Transceiver surface coils have also been used for imaging outside the brain, for example in the heart ${ }^{(32)}$ and breast imaging ${ }^{(33)}$, although quantification is extremely difficult due to inhomogeneous transmit RF fields from such coils. A more recent development for Na-MRI has seen the separation of the transmit and receive functions, with a volume resonator used for the former and a surface coil for the latter function ${ }^{(15)}$. This approach is particularly suited to applications outside the brain where detectors can be designed to optimally fit the anatomy of interest (for example, breast, kidney, etc.), thereby optimising the SNR, while the volume transmitter provides a uniform flip angle excitation which facilitates accurate quantification of the TSC. The development of phased array coils for the brain should extend these advantages to stroke imaging. For rodent brain imaging, such a dual-coil approach has been found to be essential for accurate TSC quantification ${ }^{(26)}$.

The lower SNR for sodium in vivo can be partially compensated by the use of imaging sequences with short repetition times (TR), since the longitudinal $\mathrm{T}_{1}$ relaxation time for 
sodium nuclei (measured to be $36 \mathrm{~ms}$ in the human brain at $4.7 \mathrm{~T}^{(34)}$ ) are much shorter than for hydrogen. However, a further complication for Na-MRI arises from the quadrupolar nature of the sodium nucleus due to its spin $3 / 2$ property, which due to its interaction with local electric field gradients from neighbouring nuclei in the tissue leads to a biexponential transversal $\mathrm{T}_{2}$ relaxation, with fast and slow components $\left(\mathrm{T}_{2} \sim 1-2 \mathrm{~ms}\right.$ and 15-20 $\mathrm{ms}$ in the brain, respectively) with relative proportions of $60 \%$ and $40 \%$ respectively. To acquire signal from the fast component, one must use a short echo time (TE) imaging sequence; examples of such sequences which have been used for sodium imaging in stroke include 3D projectionreconstruction (3DPR) ${ }^{(35)}$, Twisted Projection Imaging (TPI) ${ }^{(36)}$, and density-adapted 3DPR (31).

\section{CLINICAL STROKE Na-MRI STUDIES}

Acute stroke patients are often quite sick and there are significant logistical difficulties involved in carrying out sodium imaging experiments in this patient cohort, not least the maxim that "time is neurons" which makes it ethically difficult to justify the experimental imaging of patients which may postpone any available therapy options. As a result, only a handful of such studies appear in the scientific literature to date, and many patients recruited to these studies underwent sodium imaging after a thrombolytic treatment was administered, so any reperfusion effects caused by these treatments (or indeed due to spontaneous reperfusion) represent a potential confound to the results obtained. Furthermore, patient numbers on these studies have been quite low which, coupled with the inherent heterogeneity of human strokes, renders it difficult to draw definitive conclusions from existing studies.

The first study describing sodium imaging in the brain was in 1988, and although increased signal was seen in patients with herpes simplex encephalitis (due to the resulting oedema) and tumours, no signal change was detected in the single stroke patient imaged due most likely to the very poor image quality ${ }^{(37)}$. The first dedicated study of Na-MRI in stroke was reported by Shimizu et al. in 1993, who imaged 7 patients with confirmed stroke (three within 14 hours of symptom onset and the rest at 47-82 hours after onset) using a $1.5 \mathrm{~T}$ scanner ${ }^{(38)}$. The authors of this pioneering study reported no change in the sodium signal in the acute phase (that is, out to 13 hours; $n=3$ ), however a "marked signal increase" (not specified by 
the authors) was detected in the ischaemic area at 38 to 82 hours $(n=4)$. It is likely that the poor quality images achieved in this early study, due to the use of a spin echo sequence with a long TE of $13 \mathrm{~ms}$, producing significant $\mathrm{T}_{2}$ signal decay, resulted in the inability to detect any change in Na signal in the acute phase. In a study by Thulborn et al in 1999, sodium images were acquired on 42 patients with confirmed stroke, 11 of which were imaged within 24 hours of symptom onset ${ }^{(29)}$. Sodium imaging was performed on either a $1.5 \mathrm{~T}$ or $3 \mathrm{~T}$ scanner, using a dual-tuned ${ }^{23} \mathrm{Na} /{ }^{1} \mathrm{H}$ volume resonator, with all acute patients imaged using the $1.5 \mathrm{~T}$ scanner. The quantified TSC was found to be elevated in the infarcted region by at least $25 \%$ in all patients (where the infarcted regions were defined by analysis of corresponding DWI and PWI data), although no clear pattern of TSC increase could be discerned in the hours and indeed days after stroke onset. The existence of a threshold TSC value, above which irreversible tissue damage would inevitably occur even following a therapeutic intervention, was suggested by the authors who nonetheless stated that more extensive experimentation using animal models would be required to define such a threshold.

A study in 2009 succeeded in recruiting 21 stroke patients with imaging times as early as 4 hours and out to 104 hours after the known stroke onset time, with repeat scans performed on 10 of these patients ${ }^{(4)}$. All ${ }^{1} \mathrm{H}$-MRI was performed on a $1.5 \mathrm{~T}$ scanner, with the patients transferred to a $4.7 \mathrm{~T}$ system for the Na-MRI imaging, where a single-tuned resonator was used together with an imaging sequence termed "sodium projection acquisition in the steady state" (Na-PASS) to give high SNR images with 10 minute image acquisition times. Clear $\mathrm{Na}$ signal increases, relative to the contralateral side, were observed in all patients in regions exhibiting reduced water mobility (as determined from ADC maps), with a modest increase of $<10 \%$ in patients imaged within 7 hours of stroke onset $(n=5)$, followed by a more rapid increase out to $18-20$ hours, eventually reaching a plateau of $69 \pm 18 \%$ after approximately 72 hours. The regions of interest used in this analysis was presumed to represent infarcted tissue, and further increases in the relative Na signal of $76 \pm 20 \%$ were observed in follow-up scans from $88-227$ days. The ADC maps and sodium images from one patient on this study who was imaged at three time-points are presented in Figure 1, demonstrating the increase in $\mathrm{Na}$ signal (45\% increase over contralateral side) between the images acquired at 4 and 26 hours after stroke onset, with a further increase (75\% relative increase) at 91 days. 


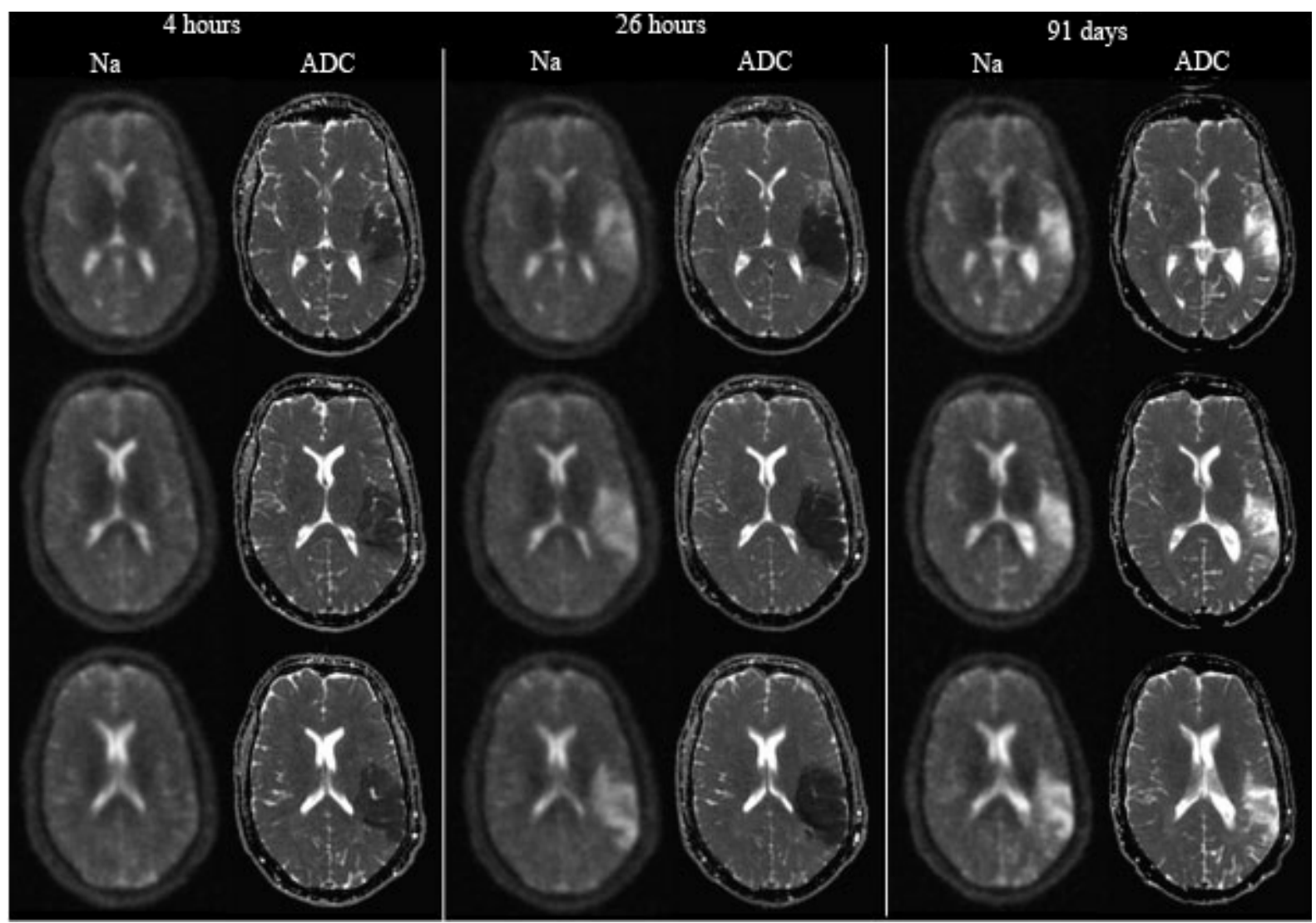

Figure 1: representative slices from a patient imaged at three time points after stroke onset, showing no increase in $\mathrm{Na}$ signal (relative to the contralateral side) at 4 hours in a region of interest drawn to correspond with the ADC-defined lesion, but with increases of $45 \%$ and $75 \%$ at the later times of 24 hours and 91 days respectively. Reproduced with permission from ${ }^{(4)}$.

The most recent human stroke study reported in the literature was carried out by Tsang et al, who aimed to investigate sodium signal changes in the PWI-DWI mismatch area during the acute stroke phase $^{(2)}$. 9 patients were imaged 4 - 32 hours after symptom onset, with followup scans performed on four, again using a $4.7 \mathrm{~T}$ scanner with a single-tuned volume resonator for the sodium imaging. In patients imaged $4-7$ hours after onset, no increase (relative to contralateral homologous tissue regions) in the sodium signal was observed in core tissue (where the region of core tissue was defined as the DWI-delineated lesion), although by 17 32 hours a significant increase in this region was measured, with an average increase of $45 \%$ $\pm 26 \%$ across the 9 patients. However, no correlation was found between this increase and the corresponding degree of hypoperfusion in the core regions. Furthermore, no change in sodium signal was detected in regions defined by the DWI-PWI mismatch in any of the patients investigated. Data from one patient in this study is presented in Figure 2, where a sodium signal increase is evident at 25.5 hours but not at 4 hours post symptom onset. 


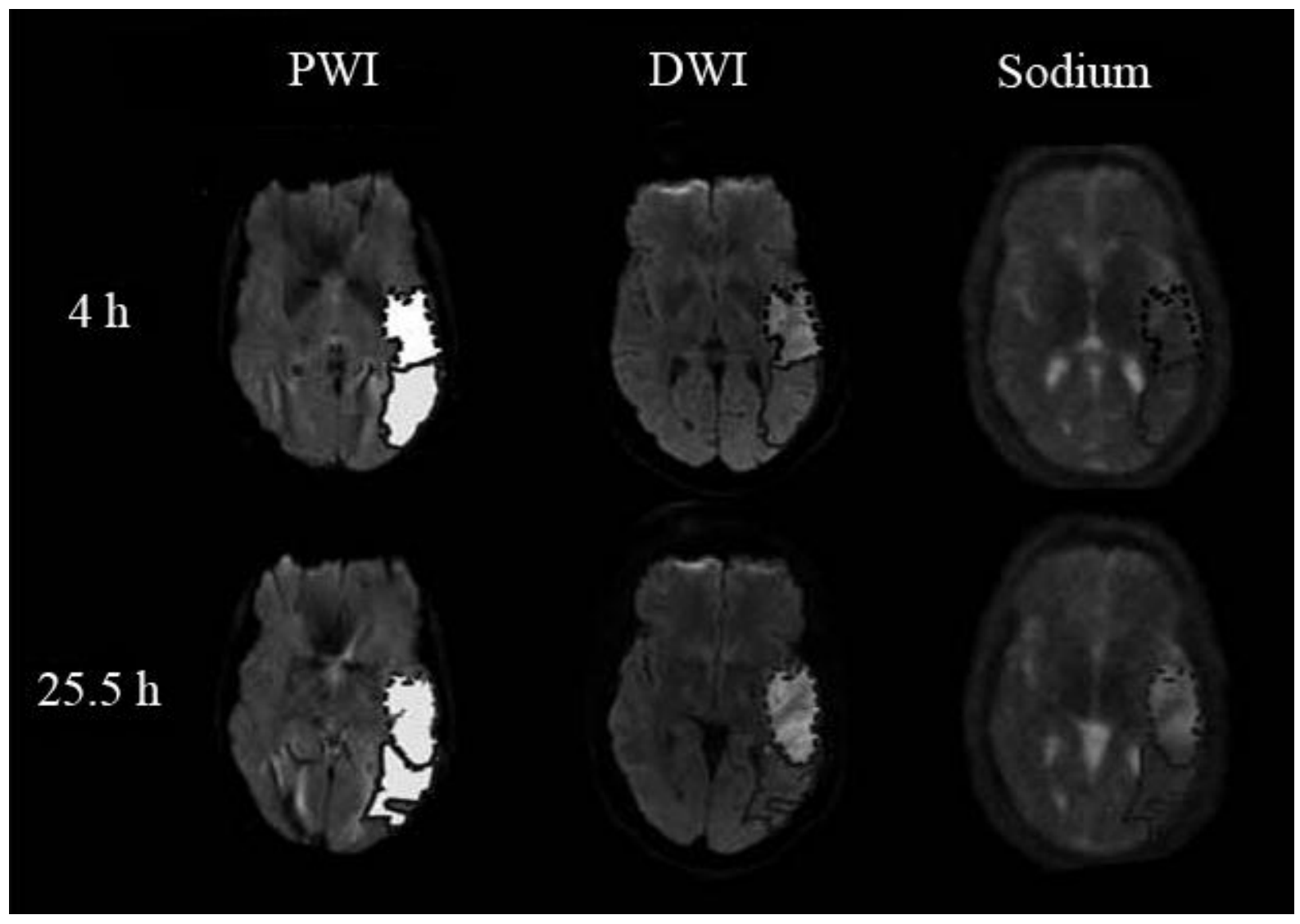

Figure 2: Perfusion, diffusion and sodium data from a representative slice of a stroke patient imaged at two time-points post symptom onset. The DWI hyperintense region is indicated by the dotted outline, with the PWI-DWI mismatch tissue by the solid outline. Note the lack of sodium signal change at 4 hours, which increased to 1.46 (relative to the contralateral side) by 25.5 hours in the core region only. Reproduced with permission from ${ }^{(2)}$.

\section{PRE-CLINICAL STROKE Na-MRI STUDIES}

The few human Na-MRI stroke studies conducted thus far suffer from a lack of temporal data on the stroke evolution, with at most two follow-up scans performed on a sub-group of patients at best, while the heterogeneity of stroke progression evident between patients makes it difficult to draw firm conclusions as to the utility of Na-MRI in the acute phase. The use of pre-clinical models of stroke have a number of advantages in this regard: the ability to control the severity and extent of the ischemic damage; precise knowledge of the stroke 
onset time; the ability to acquire temporal data in order to follow the evolution of the stroke throughout the acute phase; and the possibility to histologically verify ischemic tissue damage. The earliest experiments in this field date from 1983 in the cat ${ }^{(39)}$ and 1985 in the rat ${ }^{(40)}$, where increases in the sodium signal were detected 9 and 3 hours after the strokes were induced, respectively. However, the relatively poor image quality of these studies deterred follow-up studies for many years, with a resurgence in activity in the last decade with the appearance of papers describing studies in a variety of animals, primarily rats, rabbits and primates. In these studies, a variety of stroke models have been used, including: the intraluminal thread model ${ }^{(27,41)}$ : direct middle cerebral artery occlusion (MCAO) with combined bilateral common carotid artery occlusion (CCAO) in the rat ${ }^{(24)}$, and autologous embolic MCAO models in rabbit ${ }^{(28)}$ and non-human primate ${ }^{(42,29,3)}$. These models induced different severities of ischaemic insult, depending on the addition of CCAO, the level of collateral blood supply in the model and the neuroanatomical location of the region of interest (e.g. ischaemic core versus penumbra). Nevertheless, a common finding of these studies was a gradual increase in the sodium signal within core tissue, with rates of increase depending on the species: $\sim 22-25 \% / \mathrm{h}$ in rats, $\sim 12 \% / \mathrm{h}$ in rabbits and $\sim 5-13 \% / \mathrm{h}$ in monkeys (compared to $<\sim 5 \% / \mathrm{h}$ in humans ${ }^{(2,4,29)}$, although it should be noted that an explicit linear increase in humans has not been verified thus far). This pattern of smaller rates of increase in the larger mammals most likely reflects the increasing degree of collateral supply in their brains, a feature which has complicated the translation of findings to human studies and perhaps contributed to the failure in clinical trials of several therapies and neuroprotectants which showed promise in rodent studies.

The apparent linear increase in sodium signal measured in core tissue has lead to the idea that sodium measurements in the acute stroke period may be used to determine the stroke onset time by extrapolating back in time, with a reported accuracy of \pm 4 minutes in a rat model ${ }^{(24)}$. It was further hypothesised that there may exist a threshold TSC value in the vicinity of 70 $\mathrm{mmol} / \mathrm{L}$ above which tissue is irreversibly infarcted, but below which tissue could recover if reperfused sufficiently accompanied by a return of the TSC value to normal levels ${ }^{(29)}$. However, this hypothesis has not been verified to date; in a recent reperfusion study in a monkey model of ischemia, the initial increase in the sodium signal in the core tissue was stopped following a reperfusion of the affected brain regions, although no subsequent decrease was measured ${ }^{(42)}$. However, only three successful reperfusions were achieved, while the limited spatial and temporal data acquired may have obscured any further changes. 


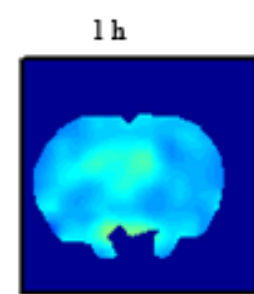

$6 \mathrm{~h}$

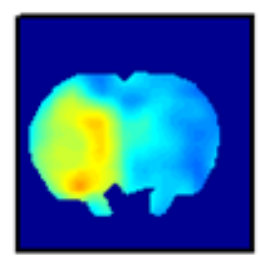

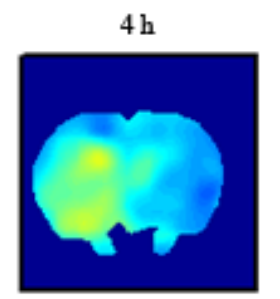

$8 \mathrm{~h}$

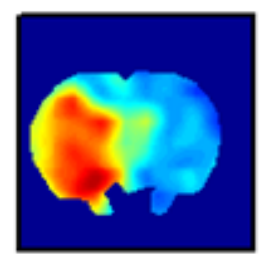

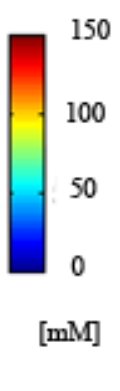

(b)

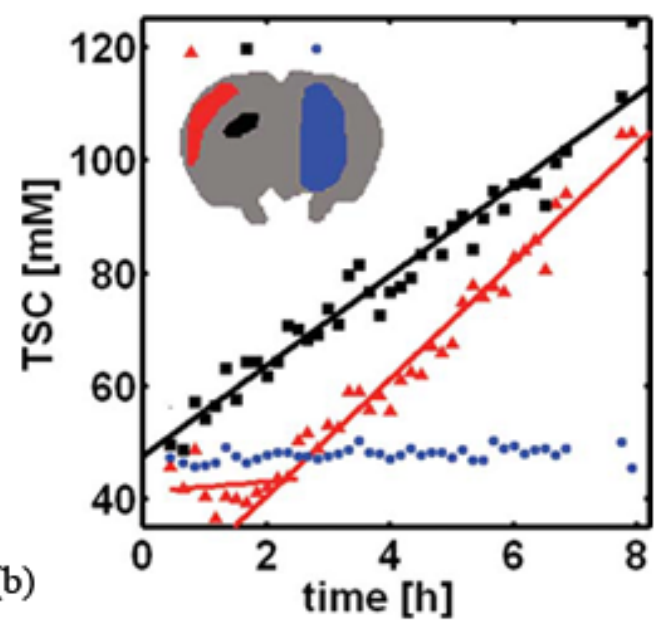

Figure 3. High spatial and temporal resolution quantified tissue sodium concentration data in a rat model of stroke. (a) Quantified TSC maps at different time-points after stroke onset from 1 slice in a representative rat brain; (b) Evolution of the quantified TSC in the hours after stroke onset at time zero, contrasting the immediate increase measured in a region located in core tissue (black) with a delayed increase in a region placed in presumed penumbra tissue (red) with a control region in the contra-lateral hemisphere (blue). Reproduced with permission from ${ }^{(27)}$.

In a recent study from my group, high temporal and spatial resolution quantified TSC data was obtained in a rat stroke model which showed regionally-disparate behaviours in the TSC increases: while the TSC increased immediately in core tissue, delays to TSC increase of up to four hours were measured in presumed penumbra tissue regions (Figure 3) ${ }^{(27)}$. In all cases, once the TSC was observed to increase, the rate of increase was the same across all areas irrespective of the delay time to increase, while areas exhibiting any increase in TSC above normal were correlated with histologically-verified infarction, suggesting that infarction of tissue occurs once the TSC increases above normal levels. Of further note in this study was a decrease of approximately $4 \mathrm{mmol} / \mathrm{L}$ measured in presumed penumbra tissue; a follow-up study where sodium, diffusion and perfusion-weighted data was correlated with histopathology, which allowed for accurate penumbra identification, detected for the first time a decrease a decrease of upwards of $20 \%$ of the contralateral sodium signal after stroke onset ${ }^{(43)}$. Representative images from one rat showing the decrease in the sodium signal in the penumbra at 0.9 and 4 hours after stroke onset are presented in Figure 4. A slight decrease in ADC values was measured in the penumbra area, above the threshold for 
infarction but nevertheless indicating that some degree of cellular oedema had occurred as a result of a perturbation of the ionic homeostasis. The intra-/extra-cellular compartmental volume shift resulting from this cell swelling may well explain this decrease in sodium signal, recalling that the measured signal represents an average across all tissue compartment volumes and hence a reduced signal would accompany a decrease in the volume of the highconcentration extra-cellular compartment. A similar decrease of $11 \pm 8 \%$ in sodium signal was detected in a rabbit model in a region which ultimately infarcted, although it was not possible to say whether this measured decrease was in core or penumbra tissue ${ }^{(28)}$. Further, a $13 \%$ decrease in sodium signal was detected in a spectroscopic study of a canine model, but again no discrimination between core and penumbra was possible ${ }^{(44)}$. Nevertheless, if verified in further studies, this decrease in sodium signal could help identify the presence of penumbra tissue in the acute phase, and hence a PWI-Na mismatch approach may be warranted, or indeed Na-MR imaging alone ${ }^{(27)}$. A further consequence of these recent findings is the potential error which will be introduced into any stroke onset time determination, via extrapolation of the sodium signal intensity back in time, in brain regions where the sodium signal may have experienced a delay or even decrease before finally increasing; rather, such an analysis must be carried out exclusively in regions which infarcted immediately or very soon after onset, information which most likely will not be available in a clinical situation, and hence the usefulness of this approach is doubtful.

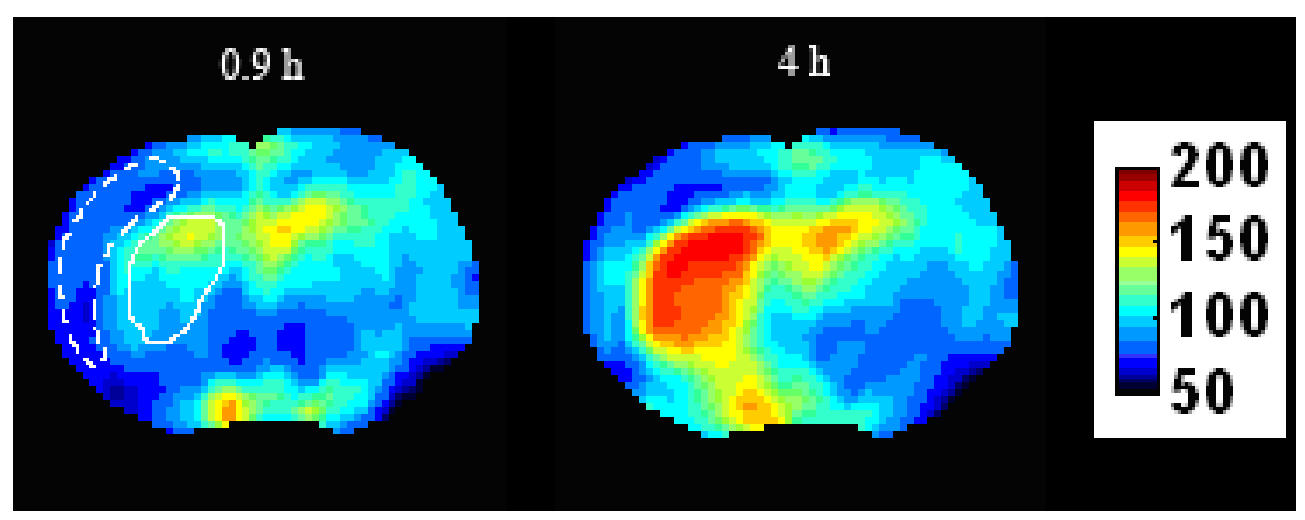

Figure 4: Sodium images from one representative stroke rat 0.9 hours and 4 hours after stroke onset, both normalised to the signal in the unaffected contra-lateral hemisphere (right side in the images). The gradual increase in sodium signal in core tissue (solid white line) is evident. However, the decrease in sodium signal in confirmed penumbra tissue (dashed white line) is clearly visible at 0.9 hours which has partially (but not fully) recovered to normal levels by 4 hours. Image generated by $\mathrm{F}$. Wetterling. 


\section{POTENTIAL OF Na-MRI IN THE ACUTE PHASE}

From an MRI perspective, the currently available techniques of PWI and DWI have not been shown to offer a definitive identification patients who will benefit from a thrombolytic treatment (some clinical trials have reported a benefit ${ }^{(45)}$, while others have not ${ }^{(46)}$ ). Thresholds of blood flow (separating infarction from benign oligemia, for example) remain uncertain, while numerous studies have shown that ADC values decrease very soon after onset time in both core and presumed penumbra tissue and remain relatively constant during the acute period, shedding little light on the evolution of the tissue damage during this critical acute period and indeed renormalizing in the sub-acute period. On the other hand, the sodium signal increases progressively above normal levels during the acute period, and recent data in both rat models and human studies suggests that this increase is restricted to core tissue. The decrease in sodium signal has been observed in animal models only thus far (only one of which was in confirmed penumbra tissue in a high SNR / spatial resolution study involving ${ }^{23} \mathrm{Na}$ and ${ }^{1} \mathrm{H}$-DWI/PWI imaging coupled with histological analyses ${ }^{(43)}$ ), while the most recent human Na-MRI study ${ }^{(2)}$ reported no change in sodium signal in what was presumed to be penumbra (delineated from the PWI-DWI mismatch). However, some doubt exists as to whether the PWI-DWI mismatch truly delineates penumbra tissue, while the limited spatial resolution of this study (nominal resolution of $2.4 \times 2.4 \times 4.8 \mathrm{~mm}^{3}$, although the actual true resolution is likely to be less due to the effects of $T_{2}$-blurring and image reconstruction of the spherical k-space data) could have resulted in a partial volume effect, whereby signal from a region containing large sodium concentration (for example, the cerebrospinal fluid, CSF) would mask any small decrease in sodium signal. It is clear that further investigations in human studies, involving higher sample numbers and improved spatial resolution, will be required to establish the presence of a similar decrease. Furthermore, although the changes in sodium signal occur within 6-8 hours after stroke onset in rats, the timescales in humans are rather longer, and hence such studies should benefit from imaging patients within a longer timeframe (perhaps out to 1-2 days) after stroke onset. If a decrease in human penumbra tissue were to be established, one could envisage a situation where only sodium imaging would be required in the acute period, avoiding the ambiguities associated with blood flow thresholds and their quantification via PWI techniques (and indeed avoiding the use of contrast agents commonly used for PWI techniques) and the PWIDWI mismatch. In this (idealised) scenario, the presence of infarcted tissue would be indicated by an increase in sodium signal above normal levels, while a decrease would reveal the presence of penumbra tissue. It is clear that further clinical studies will be required to 
verify this hypothesis, and indeed further improvements in image quality (specifically, higher SNR per unit time, allowing for improved spatial resolution) will also be required to detect such small changes, if indeed they do exist. Some recent innovations in MRI, and Na-MRI in particular, which may realise this improvement are suggested in the next section.

\section{SCOPE FOR IMPROVEMENT}

The quality of sodium images have improved dramatically since the first human stroke study back in 1993 (see comparison in Figure 5), driven primarily by developments in magnet designs allowing for higher field strengths, radiofrequency coil design and ultra-short echo time sequences. While it is impossible to predict what lies ahead with any degree of certainty, one could reasonably expect further improvements particularly considering the rapid pace of developments in this field. For example, benefits may accrue from the use of phased array coil designs for Na-MRI particularly in the brain. It can also be reasonably expected that developments in the wider MRI world will impact positively on sodium imaging, for example novel image reconstruction methods for undersampled data such as iterative reconstruction ${ }^{(47)}$ and compressed sensing ${ }^{(48)}$, acquisition schemes such as RF encoding, as demonstrated recently in sodium phantom experiments ${ }^{(49)}$, or indeed improved radiofrequency pulse designs based on optimal control theory which may allow for the selective excitation of the intra-cellular component ${ }^{(50)}$.

Many advances have been made in the design of ultra-short echo time (UTE) imaging pulse sequences specifically aimed at Na-MRI, which have increased SNR and allowed for reduced acquisition times. The first such UTE sequences developed were twisted projection imaging $(\mathrm{TPI})^{(36)}$, followed in later years by $3 \mathrm{D}$ Cones ${ }^{(51)}, 3 \mathrm{D}$ projection-reconstruction (3DPR) ${ }^{(35)}$ and most recently density-adapted 3DPR ${ }^{(31)}$ and its improved $2 \mathrm{D}$ variant ${ }^{(52)}$. Other techniques have been successfully adapted for use in sodium imaging, such as single point ramped imaging with $\mathrm{T}_{1}$ enhancement (SPRITE), where a Centric SPRITE variant was recently used to quantify the TSC in brain tumours ${ }^{(53)}$, while the Sweep Imaging with Fourier Transform (SWIFT) technique may prove useful due to its ability to acquire the entire signal from the short $\mathrm{T}_{2}$ sodium component due to its effective zero echo time ${ }^{(54)}$. This remains an active area of investigation and will hopefully lead to further innovations in the coming years. 


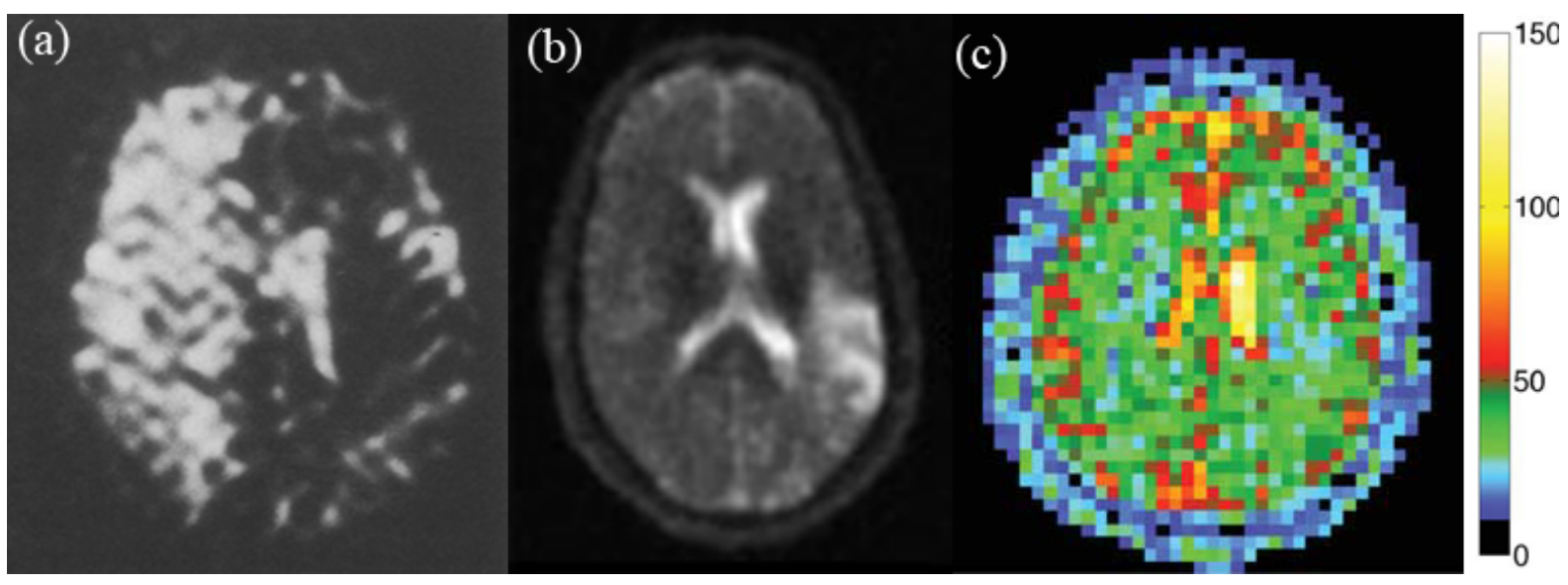

Figure 5: Illustration of the improvement in sodium image quality since the first stroke study in 1993: (a) sodium image from the first stroke study showing increased sodium signal on the right side of the brain, with spatial resolution $4 \times 4 \times 20 \mathrm{~mm}^{3}$ acquired at $1.5 \mathrm{~T}$ (reproduced with permission from ${ }^{(38)}$ ); (b) sodium image showing relative increase in signal in infarcted tissue, from a recent study at $4.7 \mathrm{~T}$, with nominal resolution $2.4 \times 2.4 \mathrm{x} 4 \mathrm{~mm}^{3}$ acquired in 10 mins (reproduced with permission from ${ }^{(4)}$ ); (c) Quantitative map of the TSC [mmol/L] acquired at $3 \mathrm{~T}$ with nominal $3.9 \mathrm{~mm}$ isotropic resolution (the true resolution was closer to $8 \mathrm{~mm}$ isotropic in brain parenchyma) acquired in 8 mins (reproduced with permission from ${ }^{(70)}$ ).

Another interesting development in the field are efforts to introduce additional contrast into sodium images via $T_{1} / T_{2}$ relaxation time or magnetisation-prepared approaches. Currently, most studies are either spin density-weighted (that is, they measure the TSC) or they use a simple $\mathrm{T}_{1}$-weighted approach (via the use of a short repetition time, TR) in order to increase the SNR per unit scanning time via signal averaging, while other short TR / flip angle combinations have been used to minimise $\mathrm{T}_{1}$-weighting with a view to optimising the achievable SNR ${ }^{(55)}$. A recent study by Nagel et al. ${ }^{(56)}$ compared these two conventional NaMRI techniques with an inversion recovery (IR)-based approach (originally introduced in $2005^{(57)}$ ) which was used to reduce the signal from oedema. The resulting heavily $\mathrm{T}_{1}$ weighted images exhibited a lower sodium signal in the muscles of patients with channelopathies which, it was hypothesised, may be due to a reduction in the $\mathrm{T}_{1}$ relaxation times in the intra-cellular compared to the extra-cellular compartments. This IR-based preparation approach was also used to improve the quantification accuracy of the bound sodium concentration in the extra-cellular space in cartilage by suppressing the free sodium signal from fluid in and around the knee in osteoarthritic patients ${ }^{(13)}$. In another study, researchers were able to differentiate between brain tumour grades using a combination of spin-density- and relaxation-weighted sodium sequences ${ }^{(58)}$. Measurements of the long $\mathrm{T}_{2}$ 
components in the brain were reported as far back as $2004^{(59)}$, although efforts to accurately quantify the short $\mathrm{T}_{2}$ component remain unsuccessful even at $7 \mathrm{~T}^{\left({ }^{(3)}\right)}$. Nevertheless, $\mathrm{T}_{2}{ }^{*}$ maps (from the long $\mathrm{T}_{2}$ component) were recently obtained in the brain using a $3 \mathrm{~T}$ scanner, albeit with an acquisition time of 60 minutes ${ }^{(60)}$. In another study using a closely-fitting surface transceiver coil, $\mathrm{T}_{2} *$ times for both the short and long $\mathrm{T}_{2}$ components (in addition to $\mathrm{T}_{1}$ times) were quantified in the breast, illustrating that such measurements are indeed possible using optimised detector coils ${ }^{(61)}$. The possibility of introducing $\mathrm{T}_{2}$ relaxation-time contrast into $\mathrm{Na}$ images may have particular relevance to stroke imaging, where the redistribution of sodium ions between the intra- and extra-cellular compartments will be accompanied by a change in $T_{2}$ values, and hence a technique capable of introducing $\mathrm{T}_{2^{-}}$ weighted contrast may well reveal more subtle effects which are presently obscured. Equally, knowledge of the changes which occur in $T_{1}$ values during the acute stroke period may also prove beneficial, if only to avoid ambiguities arising from $\mathrm{T}_{1}$-saturation effects when pushing towards minimum TR values.

There are two other existing techniques which are capable of separating, to some degree, the intra- and extra-cellular compartment signals. The first involves the use of shift reagents such as TmDOTP(5-), first introduced in $2001^{(62)}$. However, all such reagents are highly toxic to humans and it remains to be seen whether non-toxic variants can be developed. The second approach is the use of multiple quantum coherences (MQC); in tissue, the quadrupolar interaction between the spin $3 / 2$ moment of the sodium nuclei and the local electric field gradients dominate and hence triple quantum (TQ) coherences are possible. Although signal from both intra- and extra-cellular sodium ions exhibiting biexponential relaxation may pass through a TQ filter, the hope is that multiple quantum (MQ) techniques may be more selective for the intra-cellular component. In a non-human primate stroke study, the TQ sodium signal was found to increase by $226 \% \pm 70 \%$ within $0.9 \pm 0.4 \mathrm{hr}$ of the stroke onset time, while little change was observed in the single quantum (SQ) signal $(\mathrm{n}=3)$ (63). A spectroscopic study investigating the effect of glycaemia levels on single and double quantum (DQ) sodium signals in the rat forebrain in a transient ischaemia model concluded that DQ signal intensity is a more sensitive monitor of changes in intracellular sodium concentration during and after ischaemia ${ }^{(64)}$. Thus, while there is no conclusive evidence to date that one can separate the intra- from the extra-cellular components using MQ techniques, nevertheless the different evolutions in the SQ versus MQ signal in the acute period observed in some studies warrants further investigation. The main drawback with MQ techniques is the 
drastically reduced SNR, which is approximately $10 \%$ of that obtainable in conventional single quantum coherence Na-MR images, and hence this technique will benefit from an SNR boost from future technical developments. Hancu et al succeeded in acquiring TQ sodium images of the brain in 20 minutes using a 3T scanner in $1999^{(65)}$, and while problems related to $\mathrm{B}_{0}$ inhomogeneities persist several recent studies have made considerable progress in reducing their severity and increasing the $\mathrm{SNR}^{(66,67,68)}$.

In stroke imaging, the question of whether or not to quantify the TSC remains open. On the one hand, accurate knowledge of the TSC would be essential if a threshold for tissue viability were to be determined and also, for example, in patients with bilateral stroke where relative measurements (contra- to ipsi-lateral) could be insensitive to any changes (or at least unreliable). However, TSC changes due to normal ageing processes or indeed due to the presence of pathologies such as cognitive impairment, Alzheimer's disease, etc., arising perhaps due to cellular loss or shrinkage, are largely unknown (although a very recent study has looked at the effects of normal aging in the brain at $7 \mathrm{~T}^{(69)}$ ) and thus a relative comparison of the TSC in ipsi- versus contra-lateral regions may be warranted wherein the patient would act as their own control. Relative measures avoid some of the difficulties experienced with accurate TSC quantification and are certainly easier to implement in a clinical environment, although it can be expected that on-going developments in the field, for example increasing the SNR, will make quantification more robust. Already studies have demonstrated that precise and accurate quantification of TSC in the brain can be obtained in rodents (26) and humans ${ }^{(1)}$ with good spatial resolution in sub-10 minute scans using commonly-available imaging platforms for each species (a $7 \mathrm{~T}$ research system and a clinical $3 \mathrm{~T}$ scanner, respectively). However, to push the spatial resolution even further to, say, an isotropic voxel of size $6 \mathrm{~mm}$ in humans (compared to $7.5 \mathrm{~mm}$ used in ${ }^{(1)}$ ) with accurate quantification using current coil hardware designs and imaging sequences currently requires higher field strength systems (7 T and above) ${ }^{(70)}$, which are not widely available nor are they likely to be in the near future.

In conclusion, the field of Na-MRI has undergone a rapid pace of development particularly in the last decade and remains the focus of considerable research effort. It is clear that improvements in SNR will be required if Na-MRI is to play a central role in acute stroke imaging for several reasons: firstly, to detect the subtle signal changes which are likely to prove critical for penumbra tissue identification, whether via improved quantification 
accuracy or relative measurements, within a reasonable acquisition time ( $<10$ minutes); and secondly, to allow for better spatial resolution in order to minimise partial volume effects arising, for example, when regions of CSF are inadvertently included in the analysis where the three-fold more sodium concentration in CSF compared to gray or white matter will skew the data. This problem is likely to be exacerbated in aged patients where brain atrophy has occurred. Nevertheless, given the improvement in image quality achieved thus far, one can remain optimistic for the future of the field.

\section{ACKNOWLEDGEMENTS}

AJ Fagan acknowledges previous and current grant funding from Science Foundation Ireland and the Health Research Board Ireland, respectively. The author would like to thank Dr. Daniel Ryan for fruitful discussions relating to clinical stroke imaging, to the reviewers for helpful comments and suggestions.

\section{Reference List}

1. Lu AM, Atkinson IC, Claiborne TC, Damen FC, Thulborn KR: Quantitative Sodium Imaging With a Flexible Twisted Projection Pulse Sequence. Magn Reson Med 63(6), 1583-1593 (2010).

2. Tsang A, Stobbe RW, Asdaghi N et al: Relationship Between Sodium Intensity and Perfusion Deficits in Acute Ischemic Stroke. J Magn Reson Imaging 33(1), 41-47 (2011).

** The only clinical stroke study conducted to date which compares data from perfusion, diffusion and sodium imaging

3. Thulborn KR, Davis D, Snyder J, Yonas H, Kassam A: Sodium MR imaging of acute and subacute stroke for assessment of tissue viability. Neuroimaging Clin N Am 15(3), 639-53, xi (2005).

4. Hussain MS, Stobbe RW, Bhagat YA et al: Sodium Imaging Intensity Increases with Time after Human Ischemic Stroke. Ann Neurol 66(1), 55-62 (2009). 
5. Thulborn KR, Lu AM, Atkinson IC, Damen F, Villano JL: Quantitative Sodium MR Imaging and Sodium Bioscales for the Management of Brain Tumors. Neuroimaging Clinics of North America 19(4), 615-+ (2009).

6. Ouwerkerk R, Bleich KB, Gillen JS, Pomper MG, Bottomley PA: Tissue sodium concentration in human brain tumors as measured with $23 \mathrm{Na}$ MR imaging. Rad 227(2), 529-537 (2003).

7. Ouwerkerk R, Jacobs MA, Macura KL et al: Elevated tissue sodium concentration in malignant breast lesions detected with non-invasive 23Na MRI. Breast Cancer Research Treatment (2007).

8. Ouwerkerk R, Bottomley PA, Solaiyappan M et al: Tissue sodium concentration in myocardial infarction in humans: a quantitative 23Na MR imaging study. Rad 248(1), 88-96 (2008).

9. Mellon EA, Pilkinton DT, Clark CM et al: Sodium MR Imaging Detection of Mild Alzheimer Disease: Preliminary Study. Am J Neuroradiol 30(5), 978-984 (2009).

10. Inglese $\mathrm{M}$, Madelin $\mathrm{G}$, Oesingmann $\mathrm{N}$ et al: Brain tissue sodium concentration in multiple sclerosis: a sodium imaging study at 3 tesla. Brain 133, 847-857 (2010).

11. Maril N, Rosen Y, Reynolds GH, Ivanishev A, Ngo L, Lenkinski RE: Sodium MRI of the human kidney at 3 Tesla. Magn Reson Med 56, 1229-1234 (2006).

12. Haneder S, Konstandin S, Morelli JN et al: Quantitative and Qualitative (23)Na MR Imaging of the Human Kidneys at $3 \mathrm{~T}$ : Before and after a Water Load. Rad 260(3), 857-865 (2011).

13. Madelin G, Lee JS, Inati S, Jerschow A, Regatte RR: Sodium inversion recovery MRI of the knee joint in vivo at 7T. J Magn Reson 207(1), 42-52 (2010).

14. Borthakur A, Mellon E, Niyogi S, Witschey W, Kneeland JB, Reddy R: Sodium and T-1 rho MRI for molecular and diagnostic imaging of articular cartilage. NMR in Biomedicine 19(7), 781-821 (2006).

15. Wetterling F, Rennings A, Kalayciyan R, Corteville DM, Konstandin S, Schad LR: A Dual Resonator System for Whole-Body Sodium-MRI at 3T. Proc Intl Soc Mag Reson Med 471 (2011).

16. Farr TD, Wegener S: Use of magnetic resonance imaging to predict outcome after stroke: a review of experimental and clinical evidence. J Cerebr Blood F Met 30(4), 703-717 (2010).

17. Cocho D, Belvis R, Marti-Fabregas J et al: Reasons for exclusion from thrombolytic therapy following acute ischemic stroke. Neurology 64(4), 719-720 (2005).

18. Davis SM, Donnan GA, Parsons MW et al: Effects of alteplase beyond $3 \mathrm{~h}$ after stroke in the Echoplanar Imaging Thrombolytic Evaluation Trial (EPITHET): a placebo-controlled randomised trial. Lancet Neurology 7(4), 299-309 (2008). 
19. Schellinger PD, Thomalla G, Fiehler J et al: MRI-Based and CT-Based thrombolytic therapy in acute stroke within and beyond established time windows - An analysis of 1210 patients. Stroke 38(10), 2640-2645 (2007).

20. Lin SP, Song SK, Miller JP, Ackerman JJ, Neil JJ: Direct, longitudinal comparison of (1)H and (23)Na MRI after transient focal cerebral ischemia. Stroke 32(4), 925-932 (2001).

21. Miyasaka N, Kuroiwa T, Zhao FY et al: Cerebral ischemic hypoxia: Discrepancy between apparent diffusion coefficients and histologic changes in rats. Rad 215(1), 199-204 (2000).

22. Butcher K, Parsons M, Baird T et al: Perfusion thresholds in acute stroke thrombolysis. Stroke 34(9), 2159-2164 (2003).

23. Butcher KS, Parsons M, MacGregor L et al: Refining the perfusion-diffusion mismatch hypothesis. Stroke 36(6), 1153-1159 (2005).

24. Jones SC, Kharlamov A, Yanovski B et al: Stroke onset time using sodium MRI in rat focal cerebral ischemia. Stroke 37(3), 883-888 (2006).

25. Wang Y, Hu WX, Perez-Trepichio AD et al: Brain tissue sodium is a ticking clock telling time after arterial occlusion in rat focal cerebral ischemia. Stroke 31(6), 1386-1391 (2000).

26. Wetterling F, Tabbert M, Junge S, Gallagher L, Macrae IM, Fagan AJ: A doubletuned $(1) \mathrm{H} /(23) \mathrm{Na}$ dual resonator system for tissue sodium concentration measurements in the rat brain via Na-MRI. Phys Med Biol 55(24), 7681-7695 (2010).

* Discusses the use of a separate transmit-receive coil system allowing for accurate quantitative measurement of the TSC in the rat brain

27. Wetterling F, Gallagher L, Macrae IM, Junge S, Fagan AJ: Regional and Temporal Variations in Tissue Sodium Concentration During the Acute Stroke Phase. Magn Reson Med 67(3), 740-749 (2012).

** Rat brain study which revealed significant time delays before the TSC was observed to increase, with a slight decrease measures in presumed penumbra tissue, suggesting that any increase in TSC above normal levels may indicate infarction.

28. Bartha R, Lee TY, Hogan MJ et al: Sodium T2*-weighted MR imaging of acute focal cerebral ischemia in rabbits. Magn Reson Imaging 22(7), 983-991 (2004).

29. Thulborn KR, Gindin TS, Davis D, Erb P: Comprehensive MR imaging protocol for stroke management: tissue sodium concentration as a measure of tissue viability in nonhuman primate studies and in clinical studies. $\operatorname{Rad} 213(1), 156-$ 166 (1999).

* First comprehensive clinical stroke study, discusses the potential use of Na-MRI for stroke management. 
30. Fleysher L, Oesingmann N, Stoeckel B, Grossman RI, Inglese M: Sodium LongComponent T(2)* Mapping in Human Brain at 7 Tesla. Magn Reson Med 62(5), 1338-1341 (2009).

31. Nagel AM, Laun FB, Weber MA, Matthies C, Semmler W, Schad LR: Sodium MRI Using a Density-Adapted 3D Radial Acquisition Technique. Magn Reson Med 62(6), 1565-1573 (2009).

32. Sandstede JJW, Hillenbrand $\mathrm{H}$, Beer $\mathrm{M}$ et al: Time course of Na-23 signal intensity after myocardial infarction in humans. Magn Reson Med 52(3), 545-551 (2004).

33. Ouwerkerk R, Weiss RG, Bottomley PA: Measuring human cardiac tissue sodium concentrations using surface coils, adiabatic excitation, and twisted projection imaging with minimal T2 losses. J Magn Reson Imaging 21(5), 546-555 (2005).

34. Stobbe RW, Beaulieu C: Sodium relaxometry (part 2): towards the characterisation of the sodium NMR environment in the human brain using a novel relaxometry technique. Proc Intl Soc Mag Reson Med, 3104- (2006).

35. Nielles-Vallespin S, Weber MA, Bock M et al: 3D Radial Projection Technique With Ultrashort Echo Times for Sodium MRI: Clinical Applications in Human Brain and Skeletal Muscle. Magn Reson Med 57, 74-81 (2007).

36. Boada FE, Gillen JS, Shen GX, Chang SY, Thulborn KR: Fast three dimensional sodium imaging. Magn Reson Med 37(5), 706-715 (1997).

37. Grodd W, Klose U: Sodium-Mr-Imaging of the Brain - Initial Clinical-Results. Neuroradiology 30(5), 399-407 (1988).

38. Shimizu T, Naritomi H, Sawada T: Sequential-Changes on Na-23 Mri After Cerebral Infarction. Neuroradiology 35(6), 416-419 (1993).

39. Hilal SK, Maudsley AA, Simon HE et al: Invivo Nmr Imaging of Tissue Sodium in the Intact Cat Before and After Acute Cerebral Stroke. Am J Neuroradiol 4(3), 245-249 (1983).

40. Moseley ME, Chew WM, Nishimura MC et al: In vivo sodium-23 magnetic resonance surface coil imaging: Observing experimental cerebral ischemia in the rat. Magn Reson Imaging 3(4), 383-387 (1985).

41. Yushmanov VE, Kharlamov A, Yanovski B, LaVerde G, Boada FE, Jones SC: Inhomogeneous Sodium Accumulation in the Ischemic Core in Rat Focal Cerebral Ischemia by Na-23 MRI. J Magn Reson Imaging 30(1), 18-24 (2009).

42. LaVerde GC, Jungreis CA, Nemoto E, Boada FE: Sodium time course using $23 \mathrm{Na}$ MRI in reversible focal brain ischemia in the monkey. $J$ Magn Reson Imaging 30(1), 219-223 (2009). 
** Reperfusion study in a monkey model showing an arrested increase in sodium signal after reperfusion in three samples. Such studies will help identify whether a threshold for TSC exists

43. Wetterling F, Gallagher L, Holmes WM, Macrae IM, Fagan AJ: Temporal water mobility and sodium intensity measurements in penumbra and core tissue during acute stroke. Proc Intl Soc Mag Reson Med, 470- (2011).

* First study to show a decrease in sodium signal in confirmed penumbra tissue in the acute phase of a rodent model of stroke.

44. Eleff SM, Maruki Y, Monsein LH, Traystman RJ, Bryan RN, Koehler RC: Sodium, Atp, and Intracellular Ph Transients During Reversible Complete Ischemia of Dog Cerebrum. Stroke 22(2), 233-241 (1991).

45. Albers GW, Thijs VN, Wechsle L et al: Magnetic resonance imaging profiles predict clinical response to early reperfusion: The diffusion and perfusion imaging evaluation for understanding stroke evolution (DEFUSE) study. Ann Neurol 60(5), 508-517 (2006).

46. Hacke W, Furlan AJ, Al Rawi Y et al: Intravenous desmoteplase in patients with acute ischaemic stroke selected by MRI perfusion-diffusion weighted imaging or perfusion CT (DIAS-2): a prospective, randomised, double-blind, placebocontrolled study. Lancet Neurology 8(2), 141-150 (2009).

47. Block KT, Uecker M, Frahm J: Undersampled radial MRI with multiple coils. Iterative image reconstruction using a total variation constraint. Magn Reson Med 57(6), 1086-1098 (2007).

48. Lustig M, Donoho D, Pauly JM: Sparse MRI: The application of compressed sensing for rapid MR imaging. Magn Reson Med 58(6), 1182-1195 (2007).

49. Corteville DM, Wetterling F, Schad LR: RF-encoded Sodium MRI with a surface coil. Proceedings of the European Society for Magnetic Resonance in Medicine, 621- (2011).

50. Lee JS, Regatte RR, Jerschow A: Optimal control NMR differentiation between fast and slow sodium. Chemical Physics Letters 494(4-6), 331-336 (2010).

51. Gurney PT, Hargreaves BA, Nishimura DG: Design and analysis of a practical 3D cones trajectory. Magn Reson Med 55(3), 575-582 (2006).

52. Konstandin S, Nagel AM, Heiler PM, Schad LR: Two-Dimensional Radial Acquisition Technique With Density Adaption in Sodium MRI. Magn Reson Med 65(4), 1091-1097 (2011).

53. Romanzetti S, Shah NJ: In vivo quantification of Tissue Sodium Concentration in the human brain by means of a centric SPRITE sequence at 4T. Proc Intl Soc Mag Reson Med 1498 (2011).

54. Idiyatullin D, Corum C, Park JY, Garwood M: Fast and quiet MRI using a swept radiofrequency. J Magn Reson 181(2), 342-349 (2006). 
55. Stobbe R, Beaulieu C: Sodium imaging optimization under specific absorption rate constraint. Magn Reson Med 59(2), 345-355 (2008).

56. Nagel AM, Amarteifio E, Lehmann-Horn F et al: 3 Tesla Sodium Inversion Recovery Magnetic Resonance Imaging Allows for Improved Visualization of Intracellular Sodium Content Changes in Muscular Channelopathies. Investigative Radiology 46(12), 759-766 (2011).

57. Stobbe $\mathrm{R}$, Beaulieu C: In vivo sodium magnetic resonance imaging of the human brain using soft inversion recovery fluid attenuation. Magn Reson Med 54(5), 1305-1310 (2005).

58. Nagel AM, Bock M, Hartmann C et al: The Potential of Relaxation-Weighted Sodium Magnetic Resonance Imaging as Demonstrated on Brain Tumors. Investigative Radiology 46(9), 539-547 (2011).

59. Bartha $\mathrm{R}$, Menon RS: Long component time constant of Na-23 $\mathrm{T}^{*}(2)$ relaxation in healthy human brain. Magn Reson Med 52(2), 407-410 (2004).

60. Lu AM, Atkinson IC, Thulborn KR: In Vivo Brain Sodium T2* Mapping with a Multiple-echo Flexible TPI Sequence. Proc Intl Soc Mag Reson Med 3504 (2011).

61. Kaggie L, Park D, Newbould RD et al: In Vivo Breast Sodium T1 Measurements Using Inversion Recovery 3D Cones. Proc Intl Soc Mag Reson Med 3506 (2011).

62. Winter PM , Bansal N: TmDOTP(5-) as a (23)Na shift reagent for the subcutaneously implanted 9L gliosarcoma in rats. Magn Reson Med 45(3), 436-442 (2001).

63. LaVerde G, Nemoto E, Jungreis CA, Tanase C, Boada FE: Serial triple quantum sodium MRI during non-human primate focal brain ischemia. Magn Reson Med 57(1), 201-205 (2007).

64. Tyson RL, Sutherland GR, Peeling J: (23)Na nuclear magnetic resonance spectral changes during and after forebrain ischemia in hypoglycemic, normoglycemic, and hyperglycemic rats. Stroke 27(5), 957-964 (1996).

65. Hancu I, Boada FE, Shen GX: Three-dimensional triple-quantum-filtered (23)Na imaging of in vivo human brain. Magn Reson Med 42(6), 1146-1154 (1999).

66. Matthies C, Nagel AM, Schad LR, Bachert P: Reduction of B(0) inhomogeneity effects in triple-quantum-filtered sodium imaging. J Magn Reson 202(2), 239244 (2010).

67. Fleysher L, Oesingmann N, Inglese $\mathrm{M}: \mathrm{B}(0)$ inhomogeneity-insensitive triplequantum-filtered sodium imaging using a 12-step phase-cycling scheme. NMR in Biomedicine 23(10), 1191-1198 (2010).

68. Tsang A, Stobbe RW, Beaulieu C: Triple-Quantum-Filtered Sodium Imaging of the Human Brain at 4.7 T. Magn Reson Med, in press- (2012). 
69. Fleysher L, Oesingmann N, Brown RW et al: Effect of normal aging on the Intracellular Sodium Volume Fraction in the Human Brain: a 7T MRI in-vivo study. Proc Intl Soc Mag Reson Med, 589- (2011).

70. Atkinson IC, Lu AM, Thulborn KR: Clinically Constrained Optimization of flexTPI Acquisition Parameters for the Tissue Sodium Concentration Bioscale. Magn Reson Med 66(4), 1089-1099 (2011).

* Provides an in-depth discussion of the true spatial resolution obtainable at present on 3T and 9.4T platforms for quantitative TSC mapping 